BMJ Open

Sport \&

Exercise

Medicine

\section{HIIT is not superior to MICT in altering blood lipids: a systematic review and meta-analysis}

To cite: Wood G, Murrell A, van der Touw T, et al. HIIT is not superior to MICT in altering blood lipids: a systematic review and meta-analysis. BMJ Open Sport \& Exercise Medicine 2019;5:e00647. doi:10.1136/ bmjsem-2019-000647

- Additional material is published online only. To view please visit the journal online (http://dx.doi.org/10.1136/ bmjsem-2019-000647)

Accepted 29 November 2019

Check for updates

(c) Author(s) (or their employer(s)) 2019. Re-use permitted under CC BY-NC. No commercial re-use. See rights and permissions. Published by BMJ.

${ }^{1}$ School of Science and Technology, University of New England, Armidale, New South Wales, Australia

${ }^{2}$ School of Rural Medicine, University of New England, Armidale, New South Wales, Australia

Correspondence to Ms Gina Wood, School of Science and Technology, University of New England, Armidale, NSW, Australia; gwood26@myune.edu.au

\section{ABSTRACT}

Objective To compare the effects of moderate intensity continuous training (MICT) and high intensity interval training (HIIT) on adult lipid profiles; to identify training or participant characteristics that may determine exerciseinduced change in total cholesterol (TC), triglycerides (TRG), high-density lipoprotein cholesterol (HDL-C) and low-density lipoprotein cholesterol (LDL-C).

Design Systematic review and meta-analysis.

Data sources English language searches of several databases were conducted from inception until September 2019.

Eligibility criteria for excluding studies Inclusion: (1) published randomised controlled human trials with group population $n \geq 5$; (2) intervention duration $\geq 4$ weeks; (3) comparing HIIT with MICT; and (4) reporting pre-post intervention lipid measurements. Exclusion: subjects with chronic disease, $<18$ years, pregnant/lactating, in elite athletic training; and studies with a dietary or pharmaceutical intervention component.

Results Twenty-nine data sets (mmol/L) of 823 participants were pooled and analysed. Neither HIIT nor MICT was better in decreasing TC $(0.10$ ( -0.06 to 0.19$)$, $\left.\mathrm{p}=0.12, \mathrm{I}^{2}=0 \%\right)$, TRG $(-0.05(-0.11$ to 0.01$), \mathrm{p}=0.10$ $\mathrm{I}^{2}=0 \%$ ), LDL-C (0.05 ( -0.06 to 0.17$), p=0.37, \mathrm{I}^{2}=0 \%$ ), or TC/HDL-C (-0.03 (-0.36 to 0.29), $\left.p=0.85, \mathrm{I}^{2}=0 \%\right)$. HIIT significantly raised $\mathrm{HDL}-\mathrm{C}(0.07$ (0.04 to 0.11$), \mathrm{p}<0.0001$, $\left.\mathrm{I}^{2}=0 \%\right)$ compared with MICT.

Conclusion Neither HIIT nor MICT is superior for altering TC, TRG, or LDL-C, or TC-HDL-C ratio. Compared with MICT, HIIT appeared to significantly improve HDL-C. Clinicians may prescribe either protocol to encourage participation in exercise and reduce cardiovascular risk. To raise HDL-C, HIIT may result in a larger effect size compared with MICT.

PROSPERO registration number CRD42019136722.

\section{INTRODUCTION}

An abnormally elevated or lowered blood lipid profile, known as dyslipidaemia, is a significant risk factor of cardiovascular disease $(\mathrm{CVD})^{12}{ }^{2}$; ischaemic stroke $^{3}$; non-alcoholic fatty liver disease (NAFLD) ${ }^{4}$; and chronic pancreatitis. ${ }^{56}$ Dyslipidaemia frequently coexists with other Metabolic Syndrome (MetS) factors such as obesity $(\mathrm{Ob})^{7}$ and type 2 diabetes $(\mathrm{T} 2 \mathrm{D})^{89}$; and MetS is implicated in

\section{What is already known?}

- Aerobic physical activity positively impacts blood lipids, however lack of time and enjoyment are cited as impediments to exercising.

- High-intensity interval training (HIIT) appears to offer greater benefits compared with moderate intensity continuous training (MICT). Protocols are formulated to require less time spent training, however higher intensity may negatively impact enjoyment.

- Sufficient volume of aerobic physical activity is necessary to induce changes to blood lipids, however little agreement exists as to whether the shorter session duration of high-low intensity intervals or the moderate intensity of longer session steady-state exercise best changes effect size.

\section{What are the new findings?}

HIIT does not out-perform MICT in positively affecting total cholesterol (TC), triglycerides (TRG), lowdensity lipoprotein cholesterol (LDL-C) and the TC/ high-density lipoprotein cholesterol (HDL-C) ratio. However, MICT seems to be inferior to HIIT for inducing positive changes to $\mathrm{HDL}-\mathrm{C}$.

- Participant (age, gender and presence of Metabolic Syndrome (MetS) or MetS factors/risk) and intervention (weight-bearing) characteristics do appear to influence effect size.

- The multiplicity of HIIT protocols is an obstacle to endorsing a specific HIIT regime most effective for positively impacting blood lipids while accounting for time and enjoyment needs, although HIIT could be chosen in preference to MICT for improving HDL-C.

CVD risk. ${ }^{10}$ Moderate-intensity and vigorousintensity aerobic physical activity positively impacts MetS factors, thus lowering CVD risk. ${ }^{1112}$ Studies $^{1314}$ and systematic reviews ${ }^{15} 16$ have shown aerobic exercise reduces elevated total cholesterol (TC), triglycerides (TRG) and low-density lipoprotein cholesterol (LDL-C) and increases high-density lipoprotein cholesterol (HDL-C) in subclinical and clinical populations.

Much published work has examined and confirmed the beneficial physiological effects 
of aerobic physical activity or moderate intensity $(55 \%-$ $70 \%$ of maximal heart rate (MHR), rate of perceived effort (RPE) of 11-13 on the Borg scale) ${ }^{17}$ continuous training, known as MICT. The WHO recommends a minimum of 150 min per week of aerobic physical activity at moderate continuous intensity, or $75 \mathrm{~min}$ at higher intensity, to maintain or achieve health. However, WHO reports insufficient aerobic physical activity levels among adults $>18$ years. ${ }^{18}$ Poor adherence to such recommended aerobic activity or MICT protocols results from lack of time, ${ }^{19}$ and lack of support. ${ }^{20}$ Although enjoyment of exercise is positively associated with incidence of physical activity in adults, absence of enjoyment has not been significant in explaining lack of exercise, and attitudes towards exercise lack positive association with incidence of aerobic physical activity. ${ }^{21}$ Such findings have prompted searches for alternatives to MICT in order to address continuing insufficient aerobic physical activity levels.

High intensity interval training (HIIT) is a protocol of short work intervals $<60 \mathrm{~s}-8 \mathrm{~min}^{22}$ of vigorous $(70 \%-$ $90 \%$ MHR or RPE Borg scale 14-16) ${ }^{17}$ to high intensity $(\geq 90 \% \text { MHR or } \geq \text { RPE Borg scale } 17)^{17}$ interspersed with active (40\%-70\% MHR or RPE Borg scale $8-13)^{17}$ or passive (cessation of movement) recovery periods of $1-5 \mathrm{~min} .{ }^{22}$ HIIT has been employed since the mid-20th century to improve athletic exercise performance. ${ }^{22}$ Contemporary protocols developed for non-athletes are intended to reduce session time and provide a greater stimulus for physiological and psychological adaptation compared with MICT.

HIIT has been shown to increase peak oxygen consumption $\left(\mathrm{VO}_{2 \mathrm{MAX}}\right.$ or $\left.\mathrm{VO}_{2 \mathrm{PEAK}}\right)$ compared with MICT in cardiovascular disease (CVD) populations, ${ }^{23}$ despite $\mathrm{VO}_{2 \mathrm{MAX}}$ being only one component of positive changes to cardiorespiratory fitness. ${ }^{24}$ Studies indicate that a positive impact on biomedical health indices is protocol dependent in clinical ${ }^{25}$ and healthy ${ }^{26}$ populations.

To encourage individuals to undertake aerobic physical activity, both $\mathrm{HIIT}^{27}$ and MICT $^{28}$ are promoted as enjoyable and effective, although no consensus exists as to which aerobic exercise protocol is more so. Studies have shown a minimum volume of weekly aerobic exercise for a minimum duration ${ }^{29}$ and a weekly aerobic exercise energy expenditure (EEE) threshold of $1200-2200 \mathrm{kcal}^{30}$ is necessary to induce positive changes to lipids. Systematic reviews and meta-analyses of the effect of aerobic physical activity on lipid levels have established that longer intervention and session duration results in greater effects. ${ }^{31} 32$

A systematic review comparing HIIT against MICT found no difference on blood lipids in healthy and clinical populations, but no meta-analysis was conducted. ${ }^{33}$ A pooled analysis comprising only three studies and consisting of CVD, MetS and overweight populations unsurprisingly showed equivocal effects on serum lipids. ${ }^{34}$ Other systematic reviews ${ }^{163536}$ and meta-analyses ${ }^{15}$ 37-40 have investigated the effect of exercise on lipids, but have not compared HIIT against MICT. Thus no previously published meta-analysis exists that has examined the effects of HIIT versus MICT on lipids in subclinical populations.

The aim of this study was therefore to conduct a systematic review and meta-analysis comparing the effects of HIIT and MICT on TC, TRG, HDL-C, LDL-C and TC/HDL-C in subclinical populations and to examine whether one protocol surpassed the other.

\section{METHODS}

This systematic review and meta-analysis was registered in the International Prospective Register of Systematic Reviews. ${ }^{41}$ Its results are presented according to the Preferred Reporting Items for Systematic Reviews and Meta-Analyses statement. ${ }^{42}$

\section{Search strategy}

GW and NS conducted systematic English-language searches of PubMed, all EBSCO health and medical databases including SPORTDiscus, MEDLINE and CINAHL, as well as Web of Science and EMBASE from inception to September 2019.

Searches included a mix of MeSH and free text terms relevant to the concepts of: exercise training intensity for example, (high OR HIIT OR sprint OR SIT OR vigorous AND moderate continuous OR MICT OR moderate intensity continuous exercise (MICE) OR continuous moderate exercise (CME)); interval training for example, (intermittent OR interval OR reps AND training OR exercise); intervention duration for example, (weeks NOT single bout); exercise-induced lipid metabolism; metabolic syndrome for example, (metabolic syndrome OR MetS OR T2D OR diabetes OR hypertension OR overweight OR obese); and blood lipids for example, (lipids OR cholesterol OR lipoprotein OR triglycerides). Searches excluded for pregnancy, lactation, elite athletes, juveniles, CVD, stroke, cancer and NAFLD. Systematic reviews and reference lists of papers were hand searched for additional studies.

\section{Participants and interventions inclusion/exclusion criteria}

Subclinical (healthy or overweight or MetS or MetS factors such as hypertensive), and clinical (Ob and T2D) participants taking usual medications, and with a sample size population of $n \geq 5$ in HIIT and MICT groups were included.

Two distinct exercise protocols differentiated by effort as per established guidelines ${ }^{17}$ and described as either steady state (MICT) or higher effort plus active or passive recovery intervals (HIIT), separate to warm up and cool-down, were required. No restrictions were placed on exercise session time, number and time length of work and recovery intervals or exercise type. Levels and measurement of effort such as percentage of $\mathrm{VO}_{2 \text { PEAK }}$ or $\mathrm{VO}_{2 \mathrm{MAX}}$, percentage of peak heart rate $\left(\mathrm{HR}_{\mathrm{PEAK}}\right)$ or MHR or heart rate reserve or individual anaerobic threshold heart rate $\left(\mathrm{HR}_{\mathrm{IAT}}\right)$, Borg scale, metabolic equivalent (MET), or percentage of workload or watts $\left(\mathrm{W}_{\mathrm{MAX}}\right.$ or $\left.\mathrm{W}_{\mathrm{PEAK}}\right)$ were required. Resistance-training 
or combined-training interventions without separate HIIT and MICT interventions as comparators were excluded.

\section{Comparator}

HIIT protocols as the intervention were compared against MICT protocols as the control for differentiated impacts on blood lipids.

\section{Outcomes}

Pre-post intervention lipid measurements reported as $\mathrm{mmol} / \mathrm{L}$ or $\mathrm{mg} / \mathrm{dL}$ for any of TC, TRG, HDL-C, LDL-C or TC/HDL-C were required.

\section{Study selection}

GW and NS assessed the resulting titles and abstracts of randomised controlled trials (RCTs) lasting $\geq 4$ weeks, which compared HIIT and MICT protocols, and reported pre-post intervention lipid measurements in humans $\geq 18$ years. Subsequently, the full text of potentially eligible studies was reviewed according to participant, intervention and outcome inclusion and exclusion criteria. TvdT was consulted to resolve disputes. The flow of papers through the search and inclusion process is presented in figure 1 .

\section{Data extraction}

GW and AM extracted the data to a pre-established extraction form and NS and TvdT confirmed the data extraction. For each study the following information was extracted: (1) author(s), year of publication and study design characteristics, (2) demographic and clinical characteristics, (3) HIIT intervention and MICT control protocols, (4) values before and after HIIT intervention and MICT control for any of TC, TRG, HDL-C, LDL-C or TC/HDL-C ratio and expressed as mean (M) or mean difference (MD), SD or converted to SD (SE using $\mathrm{SD}=$ square root (Sample Size) x SE), as well as main findings concerning lipids.

\section{Data synthesis}

Statistical analyses were performed using Revman V.5.3 (The Nordic Cochrane Centre, Copenhagen, Denmark)

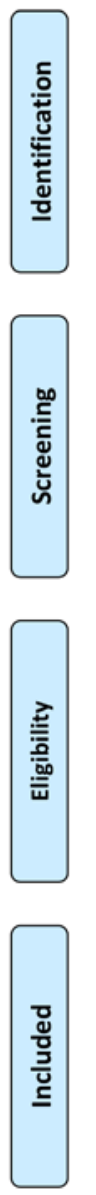

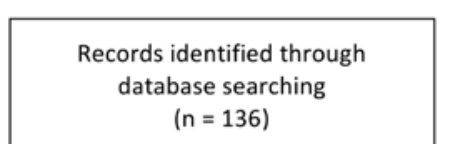
$(n=136)$

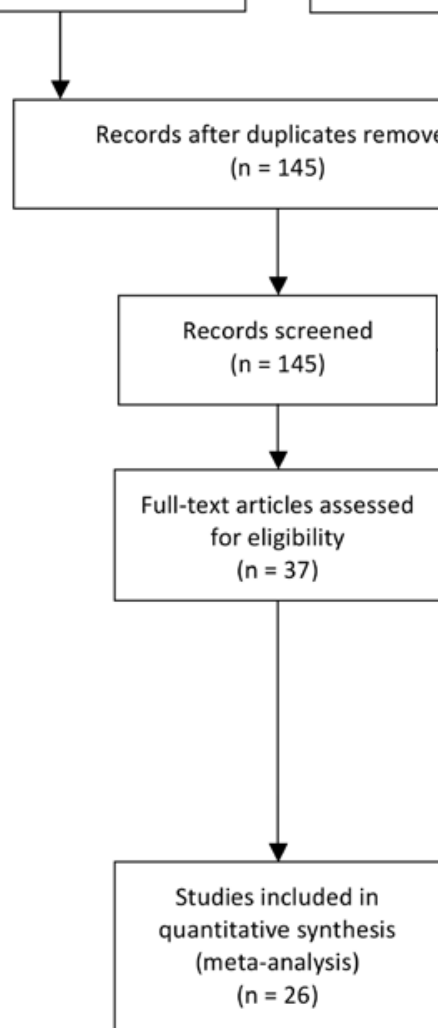

Additional records identified through other sources $(n=9)$
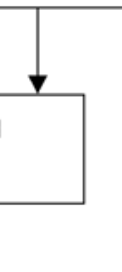
for continuous data by using the MD and SD of the MD. Where the MD and SD of the MD were not reported, the MD was calculated by subtracting the preintervention $\mathrm{M}$ from the postintervention $\mathrm{M}$. The $\mathrm{SD}$ of the $\mathrm{MD}$ was calculated as follows: $\mathrm{SD}=$ square root $\left[\left(\mathrm{SD}_{\text {pre-treatment }}\right)^{2}\right.$ $\left.+\left(\mathrm{SD}_{\text {post-treatment }}\right)^{2}-\left(2 \mathrm{r} \times \mathrm{SD}_{\text {pre-treatment }} \times \mathrm{SD}_{\text {post-treatment }}{ }^{\text {pre-treatment }}\right)\right]$, assuming a correlation coefficient $(\mathrm{r})=0.5$, considered a conservative estimate. ${ }^{43}$ Revman V.5.3 also enabled calculations of the SD of the MD using group sample size and $p$ values or $95 \%$ CIs when provided. Where data was not presented in text or tables and authors could not be reached, data presented in figures was extracted where possible.

Data were pooled for meta-analysis when two or more studies measured the same outcome and provided data in a format suitable for pooling. Where a study included multiple HIIT groups, data were entered separately for each group and the sample size of the MICT group was divided by the number of HIIT groups to eliminate inflation of the sample size. GW entered the data in Revman V.5.3; TvdT reviewed the data entry for accuracy. A random effects inverse variance model was used with the effects measure of $\mathrm{MD}$, a $5 \%$ level of significance and a $95 \% \mathrm{CI}$ to report change in outcome measures. This model was chosen to allow for different effect sizes achieved across selected studies. ${ }^{44}$

\section{Meta-analysis and subanalyses}

For meta-analysis of the four cholesterol fractions and single ratio, all included studies were grouped under each fraction and data was pooled. Subanalyses were conducted according to: age; gender; presence or absence of MetS risk and/or factor(s) or T2D; and weight-bearing or non-weight-bearing exercise.

\section{Sensitivity analysis}

In order to evaluate the influence of each study on the overall effect size of pooled data, we conducted iterative leave-one-out sensitivity analyses. ${ }^{45}$ Where subanalyses gave rise to significance, iterative leave-one-out analysis $(\mathrm{K}-1$, where $\mathrm{K}=$ the number of studies, and each study is excluded from the pool analysis one at a time) was also conducted.

\section{Heterogeneity and publication bias}

Heterogeneity was quantified using the $\mathrm{I}^{2}$ test where heterogeneity values range from $0 \%$ (homogeneity) to $100 \%$ (complete heterogeneity). ${ }^{46}$ Visual inspection of funnel plots was used to assess risk of publication bias. ${ }^{47}$ If the $95 \%$ CIs of a study were outside the pooled $95 \%$ CIs, the study was removed as an outlier. ${ }^{48}$

\section{Study quality}

Study quality was assessed by AM and GW and reviewed by NS and TvdT, using the validated Tool for the Assessment of Study Quality and Reporting in Exercise (TESTEX) ${ }^{49}$ a 15 -point scale specific to exercise training studies. A score $\geq 10$ indicates a better study quality and reporting. In the case of discrepancies NS was consulted.
A study quality subanalysis of studies grouped according to TESTEX scores $(\geq 10,<10)$ was also conducted.

\section{RESULTS}

Combined searches generated a total of 126 articles. After removal of duplicates and exclusion of articles based on abstract and title, 37 full-text articles remained for screening. One study using a non-HIIT protocol,,$^{50}$ two studies using dietary intervention, ${ }^{51}{ }^{52}$ two studies of increasing intensity not high-intensity intervals, ${ }^{13}{ }^{53}$ one study with no MICT group, ${ }^{54}$ one study reporting only preintervention values ${ }^{55}$ one study combining outcome measures of both protocols, ${ }^{56}$ and a feasibility study ${ }^{57}$ were excluded. One study tested two HIIT protocols, one of which was excluded. ${ }^{58}$ Two further excluded studies were non-RCTs. ${ }^{59}{ }^{60}$ Three studies $^{61-63}$ tested two HIIT protocols against the same group of MICT participants, hence after screening, a total of 29 data sets from 26 studies $^{2425} 5861-82$ met the stated inclusion criteria.

\section{Study, participant, and intervention characteristics}

Summarised descriptions of studies, participants and interventions included in trials are provided in Table 1 below and detailed descriptions in online supplementary file 1.

\section{Comparative outcome measures}

Total cholesterol

Twenty-one studies of 24 data sets with a total of 653 (352 HIIT, 301 MICT) subjects reported on TC MD $\left(0.10 \mathrm{mmol} / \mathrm{L}(-0.03\right.$ to 0.22$\left.), \mathrm{p}=0.12, \mathrm{I}^{2}=0 \%\right)$, shown in figure 2. No significance was found. Sensitivity analysis (K-1) did not change results.

Subanalyses did not change significance, see online supplementary files table 2 .

\section{Triglycerides}

Twenty-three studies of 25 data sets with a total of 736 (392 HIIT, 344 MICT) subjects reported on TRG MD $\left(-0.05 \mathrm{mmol} / \mathrm{L}(-0.11\right.$ to 0.01$\left.), \mathrm{p}=0.10, \mathrm{I}^{2}=0 \%\right)$, shown in figure 3. No significance was found. Sensitivity analysis (K-1) did not alter significance.

Subanalyses changed significance in favour of HIIT for (1) age grouping $35-55$ years $(-0.10 \mathrm{mmol} / \mathrm{L}(-0.19$ to -0.01 ), $\mathrm{p}=0.03, \mathrm{I}^{2}=0 \%$ ); (2) Mets or MetS factors $/$ risk $\left(-0.10 \mathrm{mmol} / \mathrm{L}(-0.18\right.$ to -0.02$\left.), \mathrm{p}=0.01, \mathrm{I}^{2}=0 \%\right)$; and (3) weight-bearing protocols $(-0.11 \mathrm{mmol} / \mathrm{L}(-0.21$ to 0.00$)$, $\left.\mathrm{p}=0.04, \mathrm{I}^{2}=0 \%\right)$. Sensitivity analysis $(\mathrm{K}-1)$ of these these subanalyses resulted in no significance with the removal of one study, ${ }^{24}$ see online supplementary files table 2.

\section{High-density lipoprotein cholesterol}

Twenty-six studies comprising 28 data sets with a total of 739 (384 HIIT, 355 MICT) subjects reported on HDL-C MD (0.07 (0.04 to 0.11), $\left.\mathrm{p}<0.0001, \mathrm{I}^{2}=0 \%\right)$, as shown in figure 4 , and favoured HIIT. Removal of one outlier ${ }^{70}$ did not alter significance. Sensitivity analysis $(\mathrm{K}-1)$ resulted in insignificance with the removal of one study, ${ }^{24}$ HDL-C 


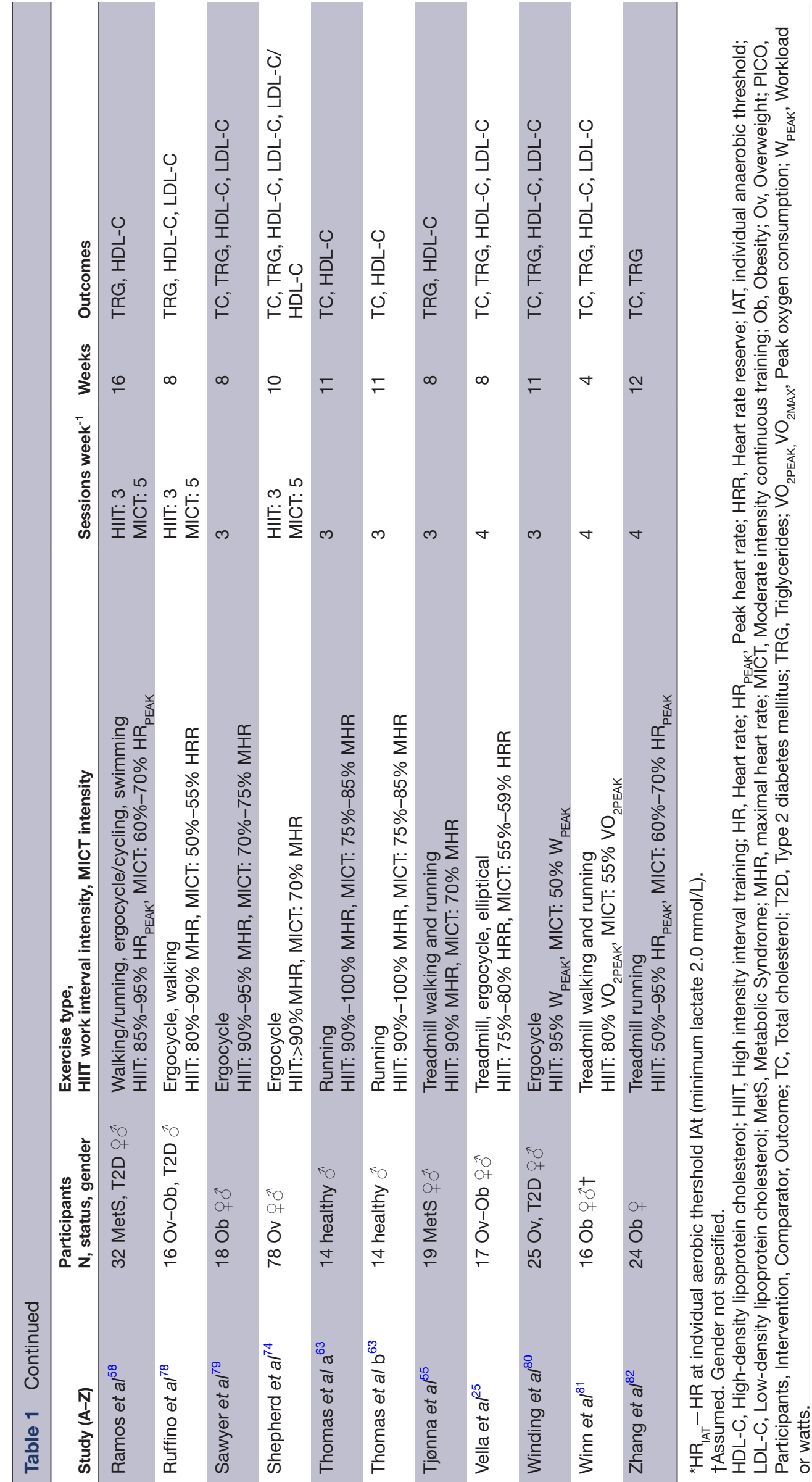




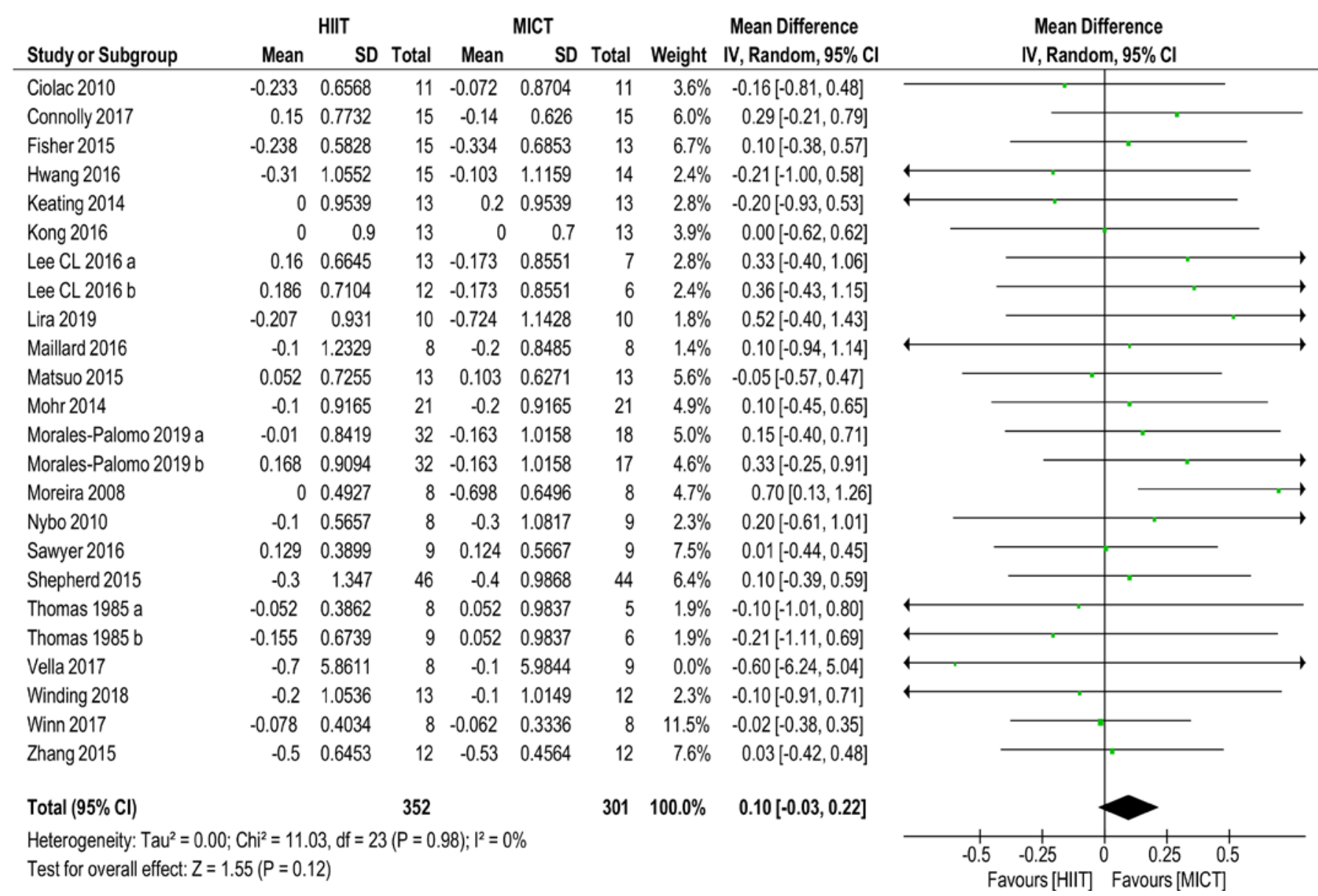

Figure 2 Total cholesterol. MD and SD expressed as mmol/L; Total = number of participants. HIIT, high intensity interval training; MICT, moderate intensity continuous training, MD, mean difference.

$\mathrm{MD}\left(0.04 \mathrm{mmol} / \mathrm{L}(0.00\right.$ to 0.08$\left.), \mathrm{p}=0.06, \mathrm{I}^{2}=0 \%\right)$, see online supplementary files table 2.

With the exception of age (all) and gender (females), subanalyses remained significant for HIIT. Applying sensitivity analysis $(\mathrm{K}-1)$ to subanalyses resulted in insignificance for the weight-bearing grouping only, see online supplementary files table 2 .

Low-density lipoprotein cholesterol

Twenty data sets of 580 (313 HIIT, 267 MICT) subjects reported on LDL-C MD (0.05 mmol/L (-0.06 to 0.17$)$, $\mathrm{p}=0.37, \mathrm{I}^{2}=0 \%$, shown in figure 5 . No significance was found. Sensitivity analysis (K-1) did not change significance.

Subanalyses did not change significance, see online supplementary files table 2 .

\section{TC/HDL-C ratio}

As shown in figure 6 , three studies with a total of 72 subjects reported on the TC/HDL-C ratio MD $(-0.03 \mathrm{mmol} / \mathrm{L}$ $(-0.36$ to 0.29$\left.), \mathrm{p}=0.85, \mathrm{I}^{2}=0 \%\right)$.

Heterogeneity and publication bias

Meta-analyses indicated zero heterogeneity for all lipid fractions, and the TC/HDL-C ratio. Visual inspection of funnel plots showed moderate-to-high likelihood of publication bias for TC and TRG, and low-to-moderate likelihood for HDL-C and LDL-C, see online supplementary files figures 2-6.

\section{Study quality and reporting}

A median TESTEX score of 11 out of 15 was obtained (range 7 to 13). TESTEX scores $(\geq 10$ or $<10)$ did not alter significance and heterogeneity, moreover sensitivity analysis (K-1) did not affect these results, see online supplementary files table 3 . No study was excluded based on its TESTEX score.

Lipid assessment

Lipid assay details are provided in online supplementary files table 4. No study was excluded based on lipid assay reporting.

\section{DISCUSSION}

\section{Meta-analysis}

This systematic review and meta-analysis aimed to compare the effects of HIIT and MICT on adult blood lipid profiles in subclinical populations and to examine whether one protocol was superior to the other. Our review is the first to include more than eight trials and compare the effect size of intermittent high-low intensity 


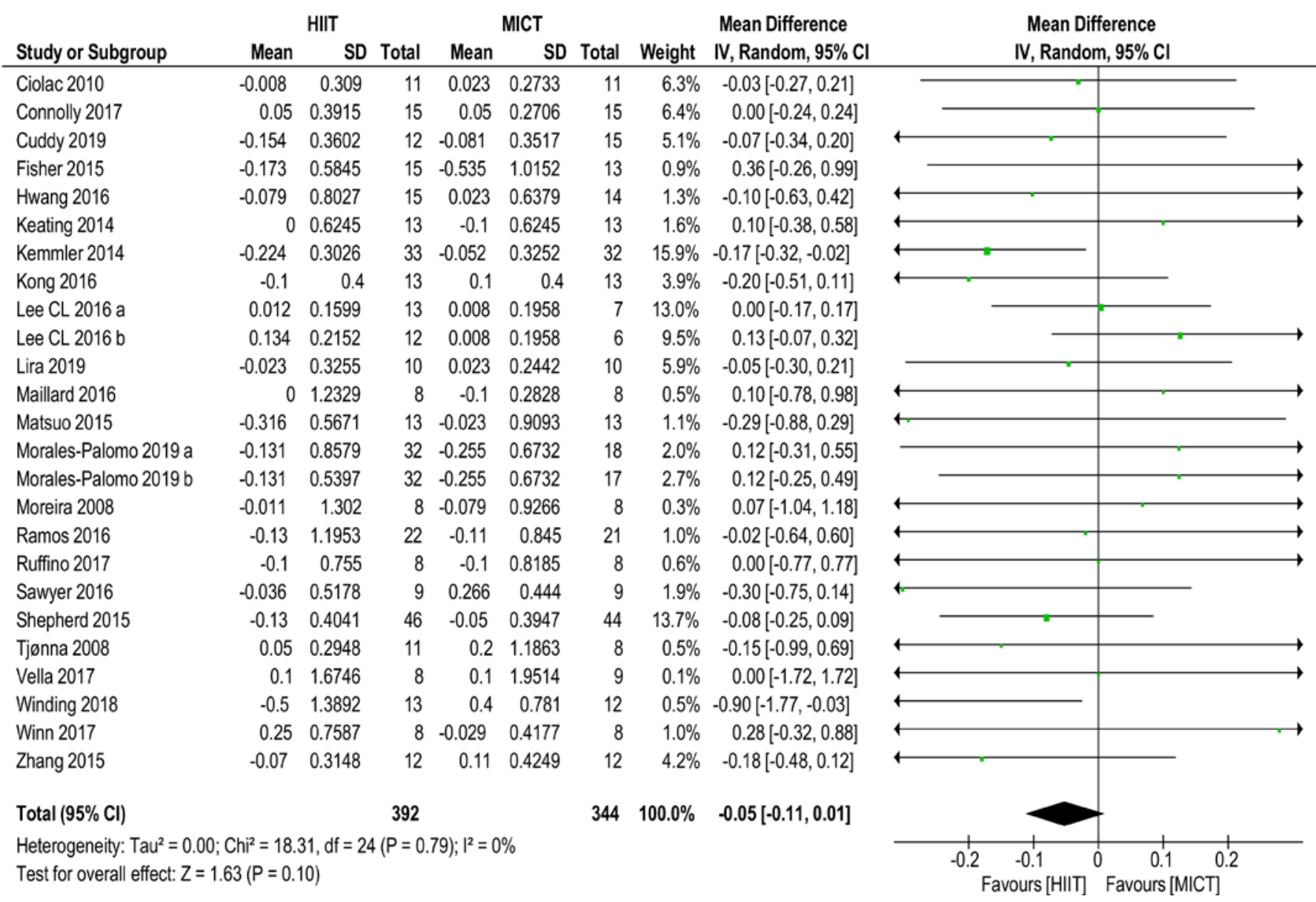

Figure 3 Triglycerides. MD and SD expressed as mmol/L; Total = number of participants. HIIT, high intensity interval training; MICT, moderate intensity continuous training, MD. mean difference.

and continuous moderate intensity in positively altering TC, TRG, HDL-C, LDL-C and the ratio of TC/HDL-C in subclinical populations. Our analysis, of 29 data sets from 26 studies, assessed the effects on lipids of weightbearing and non-weight bearing HIIT and MICT exercise therapies excluding concurrent dietary or pharmaceutical interventions. Although HIIT and MICT appear to induce positive changes, our analysis did not demonstrate that intermittent high-intensity out-performed continuous moderate-intensity protocols in achieving better lipid outcomes.

\section{Outcome measures}

Total cholesterol

We found no statistically significant evidence showing a benefit in favour of HIIT or MICT in reducing TC. Our results are similar to a previous qualitative review comparing exercise with no exercise. ${ }^{33}$ Our results differ from the findings of others ${ }^{38-40}$ whose works did not differentiate for continuous or interval protocols. We also included papers with intervention duration of 4-6 weeks; these are arguably of insufficient duration to effect change. ${ }^{33}$ MICT has been shown to prioritise fat as a primary substrate fuel in subclinical populations, ${ }^{83}$ hence it could be reasonably expected that MICT would outperform HIIT. However, a weekly energy expenditure ${ }^{30}$ or volume $\mathrm{e}^{152931}$ is required before impacts on lipids can be observed, and a number of included protocols likely fell short of this threshold. We excluded studies including dietary intervention which may have impacted our results. $^{84}$

\section{Triglycerides}

We found no difference in effect size between HIIT and MICT in positively altering TRG except for subanalyses. Our results broadly agree with a recent meta-analysis, ${ }^{85}$ although we excluded trials of cardiac patients. Our results also agree with a previous qualitative review. ${ }^{33} \mathrm{We}$ differ from the work of others, ${ }^{38-40}$ possibly because we included mixed populations or because we differentiated for protocol and intensity. A systematic review suggested TRG responded favourably to increased exercise intensity in MetS populations, ${ }^{16}$ agreeing with a previous metaanalysis, ${ }^{39}$ and our subanalysis (MetS or MetS factors/ risk) found HIIT significantly lowered TRG more than MICT.

High-density lipoprotein cholesterol

HIIT showed significance compared with MICT for affecting HDL-C, however sensitivity analysis (K-1) contradicted this result. Our findings agree with a previous meta-analysis, ${ }^{39}$ although this work compared 


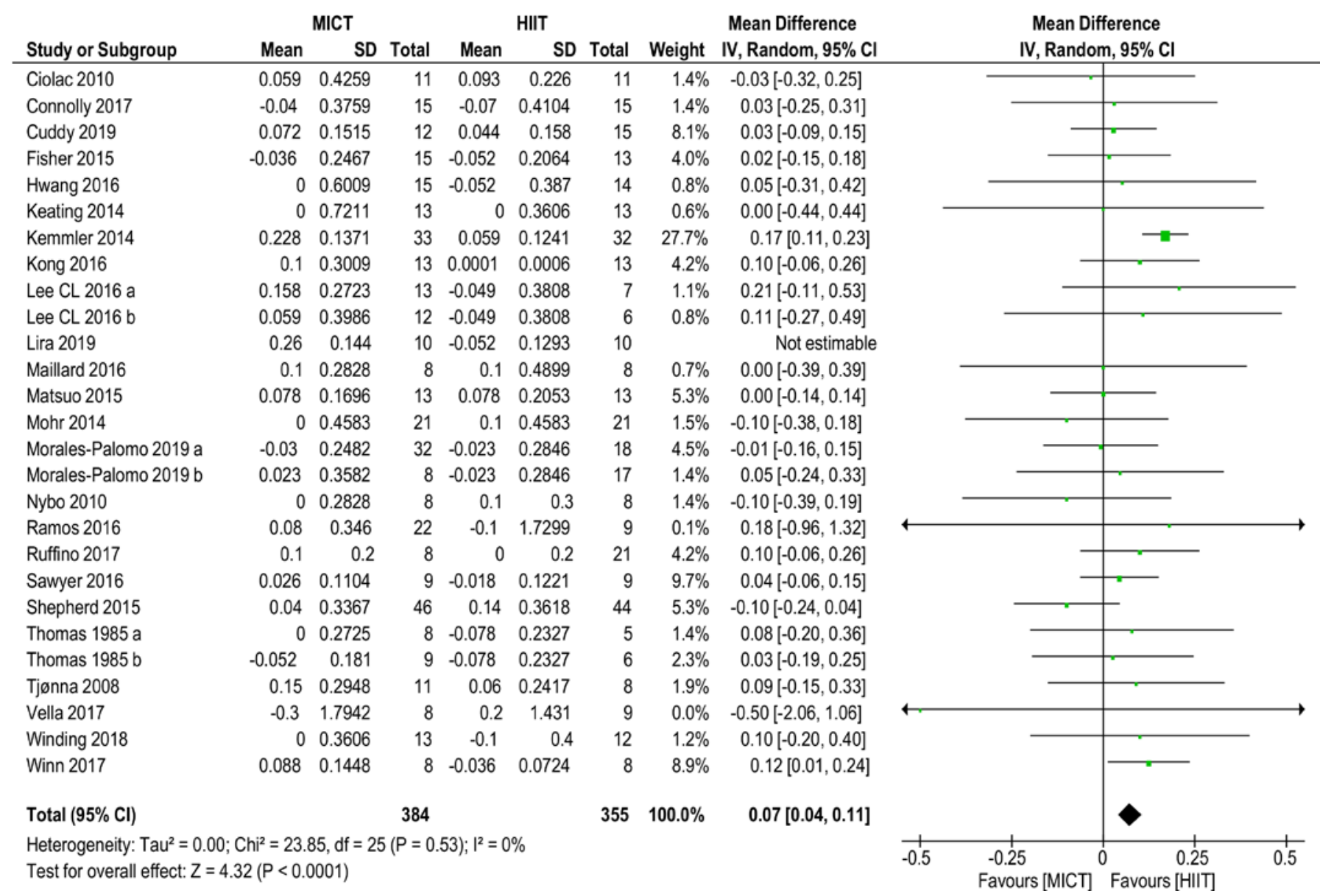

Figure 4 High density lipoprotein-cholesterol. MD and SD expressed as mmol/L; Total = number of participants. HIIT, high intensity interval training; MICT, moderate intensity continuous training, MD. mean difference.

exercise with no exercise only and focussed on overweight and obese populations. We also agree with the results of a recent meta-analysis comparing intensity, although this work focussed on studies of subjects with cardiovascular conditions. ${ }^{85}$ Our results are dissimilar to other systematic reviews, ${ }^{1633} 36$ and two (one female and one male) metaanalyses, ${ }^{38} 40$ although none of these works compared for intensity. Given the greater impact on cardiorespiratory fitness of HIIT compared with MICT, ${ }^{23}{ }^{55}$ our result is not unexpected, as HIIT would most likely outperform MICT in optimising lipid transport via an improved microvascular capillary network. However, both HIIT and MICT have been shown to equally improve muscle microvascular density. ${ }^{86}$

\section{Low-density lipoprotein cholesterol}

We found no significance for preferring HIIT to MICT for positively changing LDL-C. Our findings agree with other meta-analyses. ${ }^{35}$ We differ from two metaanalyses comparing exercise with no exercise and examining general populations, ${ }^{38} 40$ as well as a metaanalysis comparing intensity and examining LDL-C in overweight and obese populations. ${ }^{87}$ We surmise this is a corollary of our inclusion of studies with healthy participants, although our subanalyses of clinical and subclinical participants did not affect significance.
Previous work showing that LDL-C falls when accompanied by weight loss has been corroborated by a later meta-analysis comparing exercise with no exercise in overweight and obese groups. ${ }^{30}{ }^{39}$ A recent meta-analysis of HIIT compared with MICT in these populations showed no preference for either protocol in achieving weight loss. ${ }^{86} 88$ Existing higher base levels of lipids in these populations ${ }^{7}$ may have led to sufficient decrease in LDL-C to demonstrate significance for HIIT protocols. ${ }^{14}$ According to one systematic review, increasing intensity is required to impact LDL-C, ${ }^{16}$ hence MICT by its nature should have shown inferiority to HIIT. Insufficient intervention duration and probable similar overall intensity in the protocols of included studies may have obfuscated our results.

\section{TC/HDL-C ratio}

HIIT and MICT were equivalent in reducing TC/HDL-C ratio.

\section{Clinical significance and future research}

Our meta-analysis results indicate HIIT seems to be superior to MICT in affecting HDL-C. Either HIIT or MICT can be prescribed to positively affect TC, TRG, LDL-C and the TC/HDL ratio, as part of efforts to increase exercise participation to meet current aerobic physical 


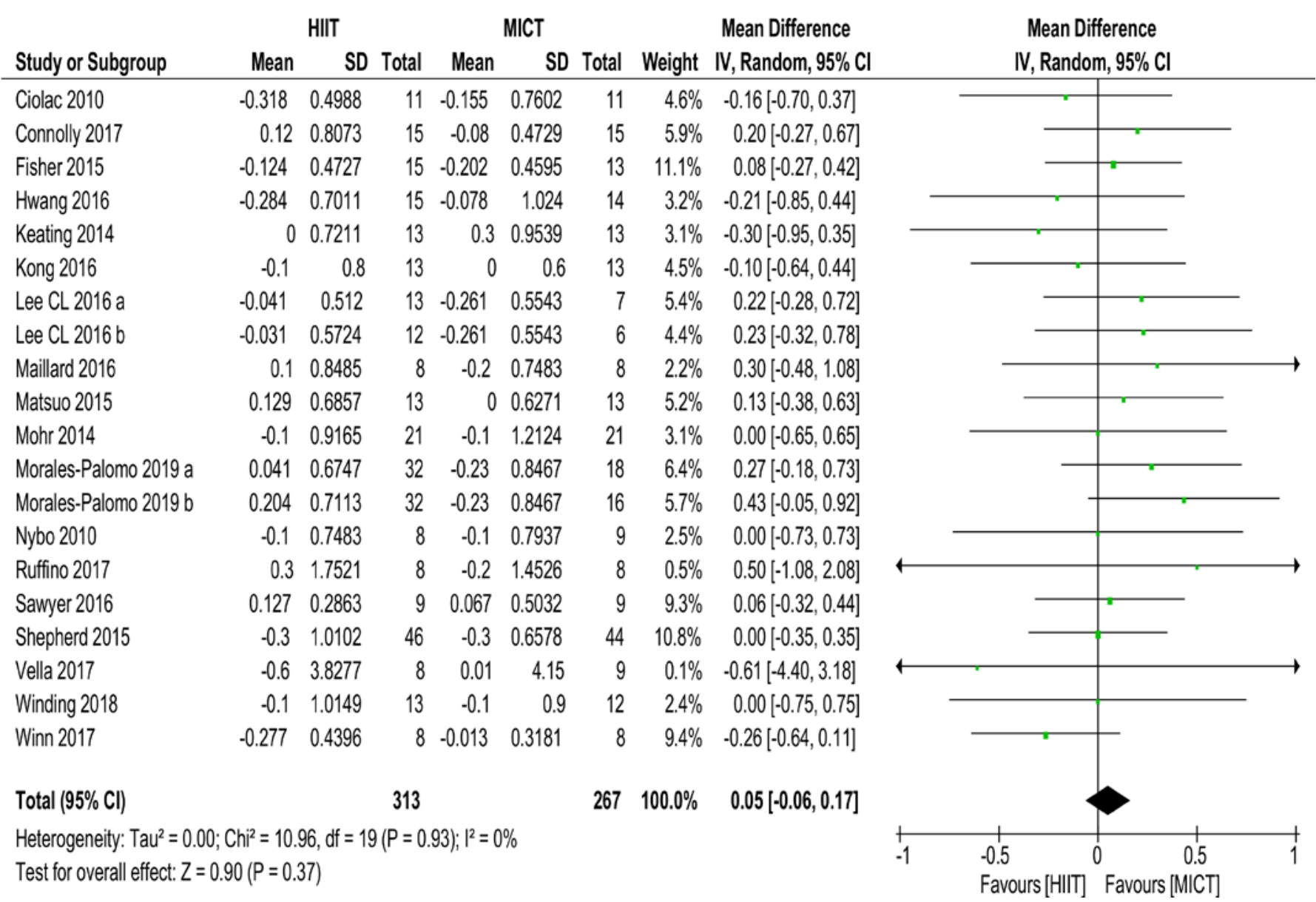

Figure 5 Low density lipoprotein-cholesterol. MD and SD expressed as mmol/L; Total = number of participants. HIIT, high intensity interval training; MICT, moderate intensity continuous training, MD. mean difference.

activity guidelines. ${ }^{18}$ Previous studies and reviews suggest a weekly minimum EEE of $>1200$ kcals and time commitment $>150$ min of aerobic physical activity at vigorous intensity is necessary to positively impact lipids. ${ }^{26} 303133$ These indicative minimum requirements exceed current weekly aerobic physical activity guidelines of $150 \mathrm{~min}$ at moderate intensity or $75 \mathrm{~min}$ at vigorous intensity. ${ }^{89}$ Sharing the results of these studies and reviews may motivate some demographics to participate in and/or increase aerobic physical activity.

Based on the number of HIIT or MICT sessions per week, our included studies generally met the minimum weekly time requirements of current aerobic physical activity guidelines. ${ }^{89}$ The EEE, effort, session duration and frequency achieved in several studies were unlikely to meet the levels required to positively impact lipids. ${ }^{26} 303133$ We propose that future research should address the following criteria to ascertain whether HIIT or MICT is better in inducing desirable changes in TC, TRG and LDL-C for varying populations: interventions should aim for duration $>8$ weeks (excluding familiarity sessions) as previously established ${ }^{31}{ }^{33}$; protocols should achieve a weekly EEE threshold $>1200$ kcals, ${ }^{30}$ or minimum session duration and frequency ${ }^{26}$; and

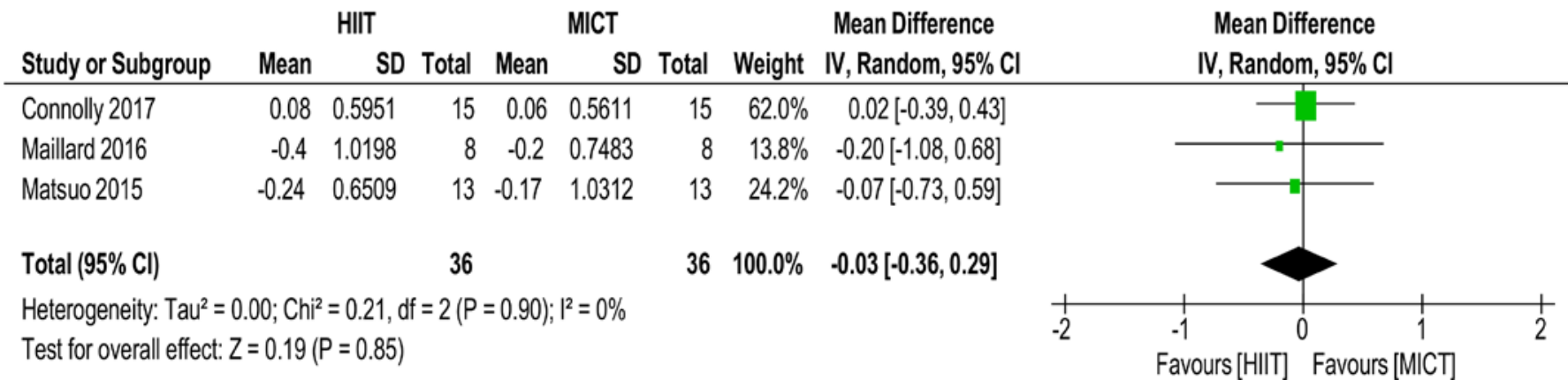

Figure $6 \mathrm{TC} / \mathrm{HDL}-\mathrm{C}$ ratio. MD and SD; Total = number of participants. HDL-C, high-density lipoprotein cholesterol; HIIT, high intensity interval training; MICT, moderate intensity continuous training, MD. mean difference; TC, total cholesterol. 
HIIT interventions should ensure that the overall effort (work:recovery ratio and repetitions) remains at or close to vigorous intensity per session, since higher intensity has been shown to impact more favourably on lipids than lower intensity. ${ }^{1326}$

\section{Strengths and limitations in the systematic review and meta-} analyses

This review has a number of strengths. To our knowledge, this review and quantitative meta-analysis is the first to compare the effects of intermittent high-intensity and continuous moderate-intensity weight-bearing and nonweight bearing protocols on cholesterol fractions and the TC/HDL-C ratio in healthy, subclinical and clinical adult populations.

Previous systematic reviews did not use the validated exercise study evaluation tool TESTEX ${ }^{48}$ to measure the quality of included studies. We followed a rigorous inclusion and exclusion protocol to ensure minimisation of confounding factors among the study populations. ${ }^{90}$

A major limitation of this review is the relatively small number of studies used in our subanalyses. This is compounded by the varying populations studied and the different exercise protocols (number and length of effort and recovery intervals, intensities, session and intervention duration, session frequency and energy expenditure) used for comparing HIIT against MICT. Some studies did not report all lipid fractions. In addition, reporting of protocol adherence and intensity used objective for example, electronic devices as well as subjective measures for example, Borg scale, self-reported $\mathrm{HR}, \log$ books, denoted by different indices of intensity (energy expenditure, $\mathrm{VO}_{2 \mathrm{MAX}}$, MHR, METs, Borg scale).

Aerobic physical activity protocols mainly consisted of running, swimming, walking, or cycling, which could have influenced results. While the majority of studies included in the analysis specified intervention duration $\geq 8$ weeks, a small number of included studies used an intervention duration of 4-6 weeks, which may have weakened results.

With respect to data pooling, we measured the difference between preintervention and postintervention means; in cases where the MD SDs were not available, we imputed the SD using pre-post SDs, $p$ values, and 95\% CIs, and hence statistical analyses depended on extrapolated data. Our imputation was conservative, and sensitivity analyses (leave-one-out) were conducted. This approach may have weakened results.

The results of our analysis may have been affected because some of the studies measured lipids as secondary and not as primary outcomes. We therefore infer that some studies were perhaps not designed with the primary goal of lipid lowering. In the paragraph on clinical significance above, we have demonstrated that earlier reviews suggest a minimum weekly EEE of $>1200$ kcals, thus some of the studies that met our inclusion criteria may have failed to meet the minimum applicable EEE, session duration and session frequency required to positively impact lipids.

\section{CONCLUSION}

Pooled analysis indicated that aerobic physical activity intensity did not influence effect size for change in TC, TRG, LDL-C and TG/HDL-C. Change in the effect size of lipids seems to be sensitive to physical activity volume rather than intensity. The exception to this appears to be HDL-C which improved more with HIIT than MICT. Our findings suggest that HIIT protocols do not confer greater improvements in lipid profiles over MICT protocols. Clinicians and allied health specialists should therefore endeavour to encourage people to undertake aerobic physical activity at or above the minimum threshold (about $1200 \mathrm{kcal}$ weekly) as a treatment or prevention strategy likely to be effective in managing lipid profiles and reducing CVD risk.

Contributors GW and NS designed the systematic review and meta-analysis, and performed searches. TvdT reviewed search results. Data extraction was performed by GW and AM. Data validation was performed by GW, AM, NS and TvdT. Data synthesis was performed by GW and AM. The article was written by GW with revisions suggested by AM, NS and TvdT.

Funding GW is supported by an Australian Government Research Training Program (RTP) Scholarship. This work received no other financial support and has no relationship to industry.

Competing interests None declared.

Patient consent for publication Not required.

Ethics approval This systematic review and meta-analysis used pooled data from previously published peer-reviewed articles for which the corresponding ethics approval was obtained by the authors of these previously published studies.

Provenance and peer review Not commissioned; externally peer reviewed. Data availability statement Data are available on request.

Open access This is an open access article distributed in accordance with the Creative Commons Attribution Non Commercial (CC BY-NC 4.0) license, which permits others to distribute, remix, adapt, build upon this work non-commercially, and license their derivative works on different terms, provided the original work is properly cited, appropriate credit is given, any changes made indicated, and the use is non-commercial. See: http://creativecommons.org/licenses/by-nc/4.0/.

\section{ORCID iD}

Gina Wood http://orcid.org/0000-0002-5096-4989

\section{REFERENCES}

1 Mora S, Cook N, Buring JE, et al. Physical activity and reduced risk of cardiovascular events: potential mediating mechanisms. Circulation 2007:116:2110-8.

2 Yusuf S, Hawken S, Ôunpuu S, et al. Effect of potentially modifiable risk factors associated with myocardial infarction in 52 countries (the INTERHEART study): case-control study. The Lancet 2004;364:937-52.

3 Goldstein LB, Adams R, Becker K, et al. Primary prevention of ischemic stroke: a statement for healthcare professionals from the stroke Council of the American heart association. Stroke 2001;32:280-99.

4 Cohen DE, Fisher EA, metabolism L. Dyslipidemia, and nonalcoholic fatty liver disease. Semin Liver Dis 2013;33:380-8.

5 Ewald N, Hardt PD, Kloer H-U. Severe hypertriglyceridemia and pancreatitis: presentation and management. Curr Opin Lipidol 2009:20:497-504.

$6 \mathrm{Ni} \mathrm{Q}$, Yun L, Xu R, et al. Correlation between blood lipid levels and chronic pancreatitis: a retrospective case-control study of 48 cases. Medicine 2014;93:e331.

7 Klop B, Elte J, Cabezas M. Dyslipidemia in obesity: mechanisms and potential targets. Nutrients 2013;5:1218-40.

8 Capurso C, Capurso A. From excess adiposity to insulin resistance: the role of free fatty acids. Vascul Pharmacol 2012;57:91-7.

9 Hill MJ, Metcalfe D, McTernan PG. Obesity and diabetes: lipids, 'nowhere to run to'. Clin Sci 2009;116:113-23. 
10 Alberti KGMM, Eckel RH, Grundy SM, et al. Harmonizing the metabolic syndrome. Circulation 2009;120:1640-5.

11 Ostman C, Smart NA, Morcos D, et al. The effect of exercise training on clinical outcomes in patients with the metabolic syndrome: a systematic review and meta-analysis. Cardiovasc Diabetol 2017; 16:110.

12 Pattyn N, Cornelissen VA, Eshghi SRT, et al. The effect of exercise on the cardiovascular risk factors constituting the metabolic syndrome: a meta-analysis of controlled trials. Sports Med 2013;43:121-33.

13 O'Donovan G, Owen A, Bird SR, et al. Changes in cardiorespiratory fitness and coronary heart disease risk factors following 24 wk of moderate- or high-intensity exercise of equal energy cost. $J$ Appl Physiol 2005;98:1619-25.

14 Greene NP, Martin SE, Crouse SF. Acute exercise and training alter blood lipid and lipoprotein profiles differently in overweight and obese men and women. Obesity 2012;20:1618-27.

15 Fikenzer K, Fikenzer S, Laufs U, et al. Effects of endurance training on serum lipids. Vascul Pharmacol 2018;101:9-20.

16 Mann S, Beedie C, Jimenez A. Differential effects of aerobic exercise, resistance training and combined exercise modalities on cholesterol and the lipid profile: review, synthesis and recommendations. Sports Med 2014;44:211-21.

17 Norton K, Norton L, Sadgrove D. Position statement on physical activity and exercise intensity terminology. J Sci Med Sport 2010:13:496-502

18 WHO. Prevalence of insufficient physical activity - Adults aged 18+ years. World Health Organization, 2019. https://www.who.int/gho/ ncd/risk factors/physical activity text/en/

19 Stutts WC. Physical activity determinants in adults. perceived benefits, barriers, and self efficacy. Aaohn J 2002;50:499-507.

20 Leslie E, Owen N, Salmon J, et al. Insufficiently active Australian College students: perceived personal, social, and environmental influences. Prev Med 1999;28:20-7.

21 Trost SG, Owen N, Bauman AE, et al. Correlates of adults' participation in physical activity: review and update. Med Sci Sports Exerc 2002;34:1996-2001.

22 Seiler S, Intervals TE. Thresholds, and long slow distance: the role of intensity and duration in endurance training. Sportscience 2019;13:32-53.

23 Hannan AL, Hing W, Simas V, et al. High-Intensity interval training versus moderate-intensity continuous training within cardiac rehabilitation: a systematic review and meta-analysis. Open Access $J$ Sports Med 2018:9:1-17.

24 Kemmler W, Scharf M, Lell M, et al. High versus moderate intensity running exercise to impact cardiometabolic risk factors: the randomized controlled RUSH-study. Biomed Res Int 2014;2014:1-10.

25 Vella CA, Taylor K, Drummer D. High-Intensity interval and moderate-intensity continuous training elicit similar enjoyment and adherence levels in overweight and obese adults. Eur J Sport Sci 2017; $17: 1203-11$

26 Johnson JL, Slentz CA, Houmard JA, et al. Exercise training amount and intensity effects on metabolic syndrome (from studies of a targeted risk reduction intervention through defined exercise). Am J Cardiol 2007;100:1759-66.

27 Heisz JJ, Tejada MGM, Paolucci EM, et al. Enjoyment for highintensity interval exercise increases during the first six weeks of training: implications for promoting exercise adherence in sedentary adults. PLoS One 2016:11:e0168534

28 Foster C, Farland CV, Guidotti F, et al. The effects of high intensity interval training vs steady state training on aerobic and anaerobic capacity. J Sports Sci Med 2015;14:747-55.

29 Kraus WE, Houmard JA, Duscha BD, et al. Effects of the amount and intensity of exercise on plasma lipoproteins. N Engl $J$ Med 2002;347:1483-92.

30 Durstine JL, Grandjean PW, Davis PG, et al. Blood lipid and lipoprotein adaptations to exercise. Sports Medicine 2001;31:1033-62.

31 Hespanhol Junior LC, Pillay JD, van Mechelen W, et al. MetaAnalyses of the effects of habitual running on indices of health in physically inactive adults. Sports Med 2015;45:1455-68.

32 Kodama Set al. Effect of aerobic exercise training on serum levels of high-density lipoprotein cholesterol. Arch Intern Med 2007;167:999-1008

33 Kessler HS, Sisson SB, Short KR. The potential for high-intensity interval training to reduce cardiometabolic disease risk. Sports Medicine 2012;42:489-509.

34 Hwang C-L, Wu Y-T, Chou C-H. Effect of aerobic interval training on exercise capacity and metabolic risk factors in people with cardiometabolic disorders: a meta-analysis. J Cardiopulm Rehabil Prev 2011;31:378-85.
35 Leon AS, Sanchez OA. Response of blood lipids to exercise training alone or combined with dietary intervention. Med Sci Sports Exerc 2001;33:S502-15.

36 Tambalis K, Panagiotakos DB, Kavouras SA, et al. Responses of blood lipids to aerobic, resistance, and combined aerobic with resistance exercise training: a systematic review of current evidence. Angiology 2009;60:614-32.

37 Halbert JA, Silagy CA, Finucane P, et al. Exercise training and blood lipids in hyperlipidemic and normolipidemic adults: a meta-analysis of randomized, controlled trials. Eur J Clin Nutr 1999;53:514-22.

38 Kelley GA, Kelley KS, Tran ZV. Aerobic exercise and lipids and lipoproteins in women: a meta-analysis of randomized controlled trials. J Womens Health 2004;13:1148-64.

39 Kelley GA, Kelley KS, Vu Tran Z. Aerobic exercise, lipids and lipoproteins in overweight and obese adults: a meta-analysis of randomized controlled trials. Int J Obes 2005;29:881-93.

40 Kelley GA, Kelley KS. Aerobic exercise and lipids and lipoproteins in men: a meta-analysis of randomized controlled trials. J Mens Health Gend 2006;3:61-70

41 Booth A, Clarke M, Dooley G, et al. The nuts and bolts of Prospero: an international prospective register of systematic reviews. Syst Rev 2012;1:2.

42 Moher D, Liberati A, Tetzlaff J, et al. Preferred reporting items for systematic reviews and meta-analyses: the PRISMA statement. BMJ 2009;339:b2535.

43 Fu R, Vandermeer B, Shamliyan T, et al. Handling Continuous Outcomes in Quantitative Synthesis [Digital. Rockville MD: Agency for Healthcare Research and Quality (US), 2008-; 2013. https://www. ncbi.nlm.nih.gov/books/NBK154408/

44 Borenstein M, Hedges LV, Higgins JPT, et al. A basic introduction to fixed-effect and random-effects models for meta-analysis. Res. Synth. Method 2010;1:97-111.

45 Higgins J, Green S. Cochrane Handbook for systematic reviews of interventions. VER 5.1.0 (updated 2011) ED. Chichester, West Sussex: Hoboken NJ John Wiley \& Sons, @ 2008.

46 Higgins JPT, Thompson SG, Deeks JJ, et al. Measuring inconsistency in meta-analyses. BMJ 2003;327:557-60.

47 Egger M, Smith GD, Schneider M, et al. Bias in meta-analysis detected by a simple, graphical test. BMJ 1997;315:629-34

48 Viechtbauer W, Cheung MW-L. Outlier and influence diagnostics for meta-analysis. Res Synth Methods 2010;1:112-25.

49 Smart NA, Waldron $\mathrm{M}$, Ismail $\mathrm{H}$, et al. Validation of a new tool for the assessment of study quality and reporting in exercise training studies: TESTEX. Int J Evid Based Healthc 2015;13:9-18.

50 Pandey A, Suskin N, Poirier P. The impact of burst exercise on cardiometabolic status of patients newly diagnosed with type 2 diabetes. Canadian Journal of Cardiology 2017;33:1645-51.

51 Ramírez-Vélez R, Tordecilla-Sanders A, Téllez-T LA, et al. Similar cardiometabolic effects of high- and moderate-intensity training among apparently healthy inactive adults: a randomized clinical trial. $J$ Transl Med 2017;15:118.

52 Wallman K, Plant LA, Rakimov B, et al. The effects of two modes of exercise on aerobic fitness and fat mass in an overweight population. Res Sports Med 2009;17:156-70.

53 Mezghanni N, Chaabouni K, Chtourou $\mathrm{H}$, et al. Effect of exercise training intensity on body composition, lipid profile, and insulin resistance in young obese women. Afr J Microbiol Res 2012;6.

54 Musa DI, Adeniran SA, Dikko AU, et al. The effect of a high-intensity interval training program on high-density lipoprotein cholesterol in young men. $J$ Strength Cond Res 2009;23:587-92.

55 Schjerve IE, Tyldum GA, Tjønna AE, et al. Both aerobic endurance and strength training programmes improve cardiovascular health in obese adults. Clin Sci 2008;115:283-93.

56 Di Blasio A, Izzicupo P, D'Angelo E, et al. Effects of patterns of walking training on metabolic health of untrained postmenopausal women. J Aging Phys Act 2014;22:482-9.

57 Lunt $\mathrm{H}$, Draper N, Marshall HC, et al. High intensity interval training in a real world setting: a randomized controlled feasibility study in overweight inactive adults, measuring change in maximal oxygen uptake. PLoS One 2014:9:e83256.

58 Ramos JS, Dalleck LC, Borrani F, et al. The effect of different volumes of high-intensity interval training on proinsulin in participants with the metabolic syndrome: a randomised trial. Diabetologia 2016;59:2308-20.

59 Elmer DJ, Laird RH, Barberio MD, et al. Inflammatory, lipid, and body composition responses to interval training or moderate aerobic training. Eur J Appl Physiol 2016;116:601-9.

60 Støa EM, Meling S, Nyhus L-K, et al. High-Intensity aerobic interval training improves aerobic fitness and $\mathrm{HbA} 1 \mathrm{c}$ among persons diagnosed with type 2 diabetes. Eur J Appl Physiol 2017;117:455-67. 
61 Lee C-L, Hsu W-C, Cheng C-F. Physiological adaptations to sprint interval training with matched exercise volume. Med Sci Sports Exerc 2017;49:86-95.

62 Morales-Palomo F, Ramirez-Jimenez M, Ortega JF, et al. Effectiveness of aerobic exercise programs for health promotion in metabolic syndrome. Med Sci Sports Exerc 2019;51:1876-83.

63 Thomas TR, Adeniran SB, Iltis PW, et al. Effects of interval and continuous running on $\mathrm{HDL}$-cholesterol, apoproteins $\mathrm{A}-1$ and $\mathrm{B}$, and LCAT. Can J Appl Sport Sci 1985;10:52-9.

64 Ciolac EG, Bocchi EA, Bortolotto LA, et al. Effects of high-intensity aerobic interval training vs. moderate exercise on hemodynamic, metabolic and neuro-humoral abnormalities of young normotensive women at high familial risk for hypertension. Hypertens Res 2010;33:836-43.

65 Connolly LJ, Bailey SJ, Krustrup P, et al. Effects of self-paced interval and continuous training on health markers in women. Eur $J$ Appl Physiol 2017;117:2281-93.

66 Cuddy T, Ramos J, Dalleck L. Reduced exertion high-intensity interval training is more effective at improving cardiorespiratory fitness and cardiometabolic health than traditional moderateintensity continuous training. Int J Environ Res Public Health 2019;16:483.

67 Hwang C-L, Yoo J-K, Kim H-K, et al. Novel all-extremity highintensity interval training improves aerobic fitness, cardiac function and insulin resistance in healthy older adults. Exp Gerontol 2016;82:112-9.

68 Keating SE, Machan EA, O'Connor HT, et al. Continuous exercise but not high intensity interval training improves fat distribution in overweight adults. J Obes 2014;834865.

69 Kong Z, Fan X, Sun S, et al. Comparison of high-intensity interval training and Moderate-to-Vigorous continuous training for cardiometabolic health and exercise enjoyment in obese young women: a randomized controlled trial. PLoS One 2016;11:e0158589.

70 Lira FS, Antunes BM, Figueiredo C, et al. Impact of 5-week highintensity interval training on indices of cardio metabolic health in men. Diabetes Metab Syndr 2019;13:1359-64.

71 Mohr M, Nordsborg NB, Lindenskov A, et al. High-Intensity intermittent swimming improves cardiovascular health status for women with mild hypertension. Biomed Res Int 2014;728289.

72 Moreira MM, HPCd S, Schwingel PA, et al. Effects of aerobic and anaerobic exercise on cardiac risk variables in overweight adults. Arq Bras Cardiol 2008;91:219-26.

73 Nybo L, Sundstrup E, Jakobsen MD, et al. High-Intensity training versus traditional exercise interventions for promoting health. Med Sci Sports Exerc 2010;42:1951-8.

74 Shepherd SO, Wilson OJ, Taylor AS, et al. Low-Volume high-intensity interval training in a Gym setting improves Cardio-Metabolic and psychological health. PLoS One 2015;10:e0139056.

75 Matsuo T, So R, Shimojo N, et al. Effect of aerobic exercise training followed by a low-calorie diet on metabolic syndrome risk factors in men. Nutr Metab Cardiovasc Dis 2015;25:832-8.

76 Fisher G, Brown AW, Bohan Brown MM, et al. High intensity Intervalvs moderate Intensity- training for improving cardiometabolic health in overweight or obese males: a randomized controlled trial. PLOS One 2015;10:e0138853.

77 Maillard F, Rousset S, Pereira B, et al. High-Intensity interval training reduces abdominal fat mass in postmenopausal women with type 2 diabetes. Diabetes Metab 2016;42:433-41.

78 Ruffino JS, Songsorn P, Haggett M, et al. A comparison of the health benefits of reduced-exertion high-intensity interval training (ReHiT) and moderate-intensity walking in type 2 diabetes patients. Appl Physiol Nutr Metab 2017;42:202-8.

79 Sawyer BJ, Tucker WJ, Bhammar DM, et al. Effects of high-intensity interval training and moderate-intensity continuous training on endothelial function and cardiometabolic risk markers in obese adults. J Appl Physiol 2016;121:279-88.

80 Winding KM, Munch GW, lepsen UW, et al. The effect on glycaemic control of low-volume high-intensity interval training versus endurance training in individuals with type 2 diabetes. Diabetes Obes Metab 2018;20:1131-9.

81 Winn NC, Liu Y, Rector RS, et al. Energy-matched moderate and high intensity exercise training improves nonalcoholic fatty liver disease risk independent of changes in body mass or abdominal adiposity - a randomized trial. Metabolism 2018;78:128-40.

82 Zhang $\mathrm{H}$, Tong T, Qui W, et al. Effect of high-intensity interval training protocol on abdominal fat reduction in overweight Chinese women: a randomized controlled trial. Kinesiology 2015;47:57-66.

83 Bircher S, Knechtle B. Relationship between fat oxidation and lactate threshold in athletes and obese women and men. J Sports Sci Med 2004:3:174-81.

84 Foster-Schubert KE, Alfano CM, Duggan CR, et al. Effect of diet and exercise, alone or combined, on weight and body composition in overweight-to-obese postmenopausal women. Obesity 2012;20:1628-38.

85 Xie B, Yan X, Cai X, et al. Effects of high-intensity interval training on aerobic capacity in cardiac patients: a systematic review with metaanalysis. Biomed Res Int 2017;2017:1-16.

86 Cocks M, Shaw CS, Shepherd SO, et al. Sprint interval and moderate-intensity continuous training have equal benefits on aerobic capacity, insulin sensitivity, muscle capillarisation and endothelial eNOS/NAD(P)Hoxidase protein ratio in obese men. $J$ Physiol 2016;594:2307-21.

87 Su L, Fu J, Sun S, et al. Effects of HIIT and MICT on cardiovascular risk factors in adults with overweight and/or obesity: a metaanalysis. PLoS One 2019;14:e0210644.

88 Wewege M, van den Berg R, Ward RE, et al. The effects of highintensity interval training vs. moderate-intensity continuous training on body composition in overweight and obese adults: a systematic review and meta-analysis. Obesity Reviews 2017;18:635-46.

89 Brown W, Bauman A, Bull F, et al. Development of evidence-based physical activity recommendations for adults (18-64 years). in: health, editor. Canberra: Commonwealth of Australia, 2013: 161.

90 Berman NG, Parker RA. Meta-Analysis: neither quick nor easy. BMC Med Res Methodol 2002;2:10. 